\title{
Efficacité du ramassage et du brûlage systématiques des bois morts dans la lutte contre le foreur de bois, Apate terebrans, dans les anacarderaies du Nord- Bénin
}

\author{
Alexis Onzo ${ }^{1 *}$, Jeanine T. Biaou ${ }^{1}$, Cyriaque Agboton ${ }^{2}$ \\ ${ }^{1}$ Faculté d'Agronomie, Département des Sciences et Techniques de Production Végétale, Université de Parakou, \\ B.P. 123, Parakou, Bénin, Afrique de l'Ouest \\ 2International Institute of Tropical Agriculture, 08 B.P. 0932, Cotonou, Benin, West Africa \\ * Auteur correspondant: E-mail: onzalex@yahoo.com or alexisonzo@gmail.com; Adresse postale: Faculté \\ d’Agronomie, Université de Parakou, B.P. 123, Parakou, République du Bénin (Afrique de l'Ouest)
}

Original submitted in on $6^{\text {th }}$ November 2017. Published online at www.m.elewa.org on $31^{\text {st }}$ January 2018 https://dx.doi.org/10.4314/jab.v121i1.7

\section{RESUME}

Objectifs : La présente étude vise à réduire de façon écologique l'infestation des anacarderaies du NordBénin par le foreur de bois, Apate terebrans, un coléoptère ravageur de l'anacardier en Afrique de l'Ouest.

Méthodologie et résultats: Les travaux ont été conduits d'août 2015 à mars 2016 à N'dali, Parakou et Tchaourou, trois communes du Nord-Bénin. Par commune deux vergers ont été sélectionnés dont le premier comme témoin tandis que le second a été soumis à des mesures prophylactiques, notamment, le ramassage-brûlage systématique des bois morts à l'intérieur comme aux alentours du verger. Les données collectées sont le taux d'infestation des anacarderaies et la densité par arbre de $A$. terebrans. Des résultats des essais, il ressort que les taux d'infestation des anacarderaies par $A$. terebrans ainsi que les densités actuelles de $A$. terebrans par anacardier ont été significativement plus élevées dans les plantations témoins (respectivement $60,0 \pm 9,0 \%$ et 10,73 $\pm 2,10$ trous/arbre) que dans celles soumises au ramassage-brûlage (respectivement $43,0 \pm 9,0 \%$ et $6,83 \pm 2,10$ trous/arbre).

Conclusion et application des résultats: II ressort de cette étude que les anacarderaies soumises au ramassage-brûlage systématiques des bois morts ont été moins attaquées par le foreur. Une application plus généralisée et à long terme de cette pratique réduira remarquablement la pression de $A$. terebrans dans les anacarderaies, pour une augmentation quantitative et qualitative de leur productivité.

Mots-clés : Anacardium occidentale, Insectes foreurs de bois, Bostrichidae, Mesures prophylactiques, Noix de cajou. 
Onzo et al., J. Appl. Biosci. 2018 Efficacité du ramassage et du brûlage systématiques des bois morts dans la lutte contre le foreur de bois, Apate terebrans, dans les anacarderaies du Nord-Bénin

Dead-wood collection and burning: an effective control measure against the wood borer Apate terebrans in cashew orchards in Northern-Benin

\section{ABSTRACT}

Objectives: This study aims at reducing, using a simple and ecological sustainable approach, the increasing infestation of cashew orchards in Northern-Benin by the wood borer Apate terebrans, an important cashew pest in West Africa.

Methodology and results: The study was conducted from august 2015 to march 2016 at N'dali, Parakou and Tchaourou, three municipalities of Northern-Benin. Two orchards were selected in each municipality and one of them was subject to a systematic collection and burning of dead woods within and around the orchard whereas the second was without any special measures and served as control. Data collected included the infestation rate of cashew orchards and $A$. terebrans density per tree. Both the infestation rates of cashew orchards and current $A$. terebrans densities were significantly higher in the control treatment $(60.0 \pm 9.0 \%$ and $10.73 \pm 2.10$ holes/tree, respectively), than in the dead-wood collection and burning treatment $(43.0 \pm 9.0 \%$ and $6.83 \pm 2.10$ entry holes/tree, respectively).

Conclusions and application of findings: It appears from this study that cashew orchards subject to systematic collection-burning of dead wood were less attacked by the wood borer. A widespread and longterm application of these prophylactic measures will significantly reduce $A$. terebrans densities in cashew orchards, thereby significantly increasing their productivity and the quality of the cashew nuts produced.

Keywords: Anacardium occidentale, cashew wood borer, Bostrichidae, prophylactic measures, cashew nut.

\section{INTRODUCTION}

La noix d'anacarde ou noix de cajou (Anacardium occidentale L.) représente la deuxième culture d'exportation au Bénin après le coton (Gagnon, 1998), et joue désormais un grand rôle dans l'économie Béninoise. La noix de cajou d'origine Béninoise est très recherchée sur le marché mondial à cause de sa qualité car dépourvue de résidus de pesticides (FAO, 2002). Cette production biologique se trouve, cependant, confrontée à une baisse de productivité des anacarderaies due à un certain nombre de contraintes phytosanitaires. En effet, de récentes études ont montré que l'anacardier au Bénin (Ouessou, 2010; Afouda et al., 2013 ; Agboton et al., 2014 ; Agboton et al., 2017 ; Agboton et al., 2018) et dans la sous-région ouest-Africaine (Dwomoh et al., 2008; Asogwa et al., 2009; Vasconcelos et al., 2014) est attaqué par de nombreux maladies et insectes ravageurs. Parmi les ravageurs, les plus importants en terme de dégâts et de fréquence sont, notamment : le foreur de bois Apate terebrans Pallas (Coleoptera : Bostrichidae), la mineuse de feuille Eteoryctis gemoniella Stainton (Lepidoptera: Gracillariidae) et une diversité de punaises dont les plus dommageables appartiennent au genre Helopeltis (Hemiptera : Miridae). Le rapport de PADSE (2004) place $A$. terebrans au rang des ravageurs les plus redoutables de l'anacardier au Bénin. Ce foreur de bois affecte considérablement le développement et la productivité des plantations matures en forant, à l'intérieur du tronc et des branches de l'arbre, des galeries dans lesquelles il se nourrit, rejetant au pied des arbres attaqués une forte quantité de sciures (Ouessou, 2010). L'adulte de $A$. terebrans, de couleur noire, de forme allongée et presque cylindrique, a une tête dirigée vers le bas et recouverte dorsalement par le thorax. La femelle se distingue du mâle par une sorte de grande touffe de cheveux jaunes avec deux saillies triangulaires sur le front. La larve a un corps de couleur jaunâtre, doté de trois paires de pattes thoraciques (Agboton et al., 2014). L'éclosion de l'œuf, le développement larvaire ainsi que la pupaison ont généralement lieu à l'intérieur du bois mort (Gardner 1957; C. Agboton, communication personnelle). Agboton et al. (2017), estiment qu'au Bénin, il n'y aurait qu'une seule génération de $A$. terebrans par an. Les attaques du ravageur commencent le plus souvent à partir des 
bordures de l'anacarderaie, sans distinction de la variété d'anacardier. L'infestation de l'anacardier par A. terebrans démarre juste au-dessus du collet de l'arbre pour atteindre les branches latérales et même les rameaux (Agboton et al., 2014). Les trous d'entrée ainsi forés suivent une même orientation et sont presque équidistants le long de la branche ou du tronc de l'arbre attaqué. Ces trous d'entrée sont de différents types suivant leur âge et l'état des galeries dans lesquelles ils débouchent (Agboton et al., 2017). Ainsi, on y distingue: des 'galeries vivantes', matérialisées extérieurement par des trous d'attaque fraîche desquels sortent encore de la sciure de bois ; des 'galeries mortes' visiblement récentes mais desquelles l'activité de l'insecte foreur n'est plus perceptible et qui sont caractérisées extérieurement par des trous de couleur noire, non encore cicatrisés; des 'galeries anciennes' qui se distinguent extérieurement des précédentes par des trous noirs déjà refermés et présentant de cicatrices très caractéristiques de l'action antérieure de A. terebrans (Agboton et al., 2017). Lorsque les attaques sont sévères, elles affaiblissent l'arbre, aboutissant fréquemment à une réduction de la production consécutivement à la rupture des branches, et quelques fois à la mort de l'arbre (Wagner et al., 2008). Pour pallier aux dégâts causés par ce ravageur, plusieurs méthodes de lutte ont été recommandées, testées

\section{MATERIEL ET METHODES}

Sites expérimentaux : Les travaux ont été conduits au Nord-Bénin dans les communes de N'dali $\left(9^{\circ} 51^{\prime} \mathrm{N}\right.$; $\left.2^{\circ} 43^{\prime} \mathrm{E}\right)$, Parakou $\left(9^{\circ} 21^{\prime} \mathrm{N}\right.$; $\left.2^{\circ} 36^{\prime} \mathrm{E}\right)$ et Tchaourou $\left(8^{\circ} 52^{\prime} \mathrm{N} ; 2^{\circ} 36^{\prime} \mathrm{E}\right)$, principales zones productrices des noix de cajou dans le département du Borgou. Cette zone est caractérisée par une saison des pluies allant d'Avril à fin Octobre, alternée d'une saison sèche allant de Novembre à Mars, période au cours de laquelle la production de la noix de cajou a lieu.

Dispositif expérimental: Au total, six plantations matures (i.e. en production) ont été sélectionnées à raison de deux plantations par commune. Les plantations retenues sont âgées de 6 à 9 ans, couvrant une superficie d'au moins 1 ha (soit un minimum de 100 arbres) et présentant des symptômes de dégâts antérieurs causés par $A$. terebrans. Les deux ou envisagées. Ainsi, au Bénin, certains producteurs se servent des rayons de vélo qu'ils insèrent dans les trous forés dans les arbres pour tuer le ravageur tandis que d'autres y pulvérisent des insecticides ou y insèrent du tampon de coton imbibé d'insecticide (Topper, 2002 ; Agboton et al., 2014). Cependant, les infestations de $A$. terebrans dans les anacarderaies ne font que croître d'une année à une autre. Ainsi, en se basant sur le cycle biologique du ravageur, Agboton et al. (2017) comme d'autres auteurs (Wagner et al., 2008), estiment qu'une gestion appropriée des vergers d'anacardiers et de leurs alentours à travers le ramassage et le brûlage systématiques des branches et troncs d'arbres morts (de l'anacardier et des autres plantes-hôtes du coléoptère) permettrait d'éliminer la majeure proportion de la source locale d'infestation et contribuerait ainsi à réduire les densités et les dégâts du ravageur dans les plantations. Cette technique a longtemps été suggérée (Wagner et al., 2008) mais n'a jamais été expérimentée scientifiquement. L'objectif global de la présente étude est donc d'évaluer l'impact du ramassage et du brûlage systématiques des branches et troncs d'arbres morts au sein et aux alentours des anacarderaies, sur la réduction des populations de $A$. terebrans et des dégâts causés aux arbres ainsi que sur l'amélioration de la productivité de ces anacarderaies.

plantations retenues par commune sont distantes d'au moins $10 \mathrm{~km}$. Au sein de chaque plantation, les mauvaises herbes ont été fauchées deux à trois fois avant que le verger n'entre en production; ceci, afin d'éviter la concurrence avec les mauvaises herbes et faciliter le ramassage des fruits. Une bande pare-feux de $10 \mathrm{~m}$ de large a été ouverte tout autour des vergers afin de prévenir les feux de brousse qui sont préjudiciables à la croissance et à la productivité des plantations. Dans chaque site les observations ont été faites dans deux vergers d'anacardiers dont l'un est soumis aux mesures prophylactiques tandis que le second n'a été soumis à aucune mesure prophylactique. La mise en place des mesures prophylactiques a consisté à sectionner les branches mortes et les rejets encombrants pour favoriser une 

dans la lutte contre le foreur de bois, Apate terebrans, dans les anacarderaies du Nord-Bénin

bonne reprise végétative. Toutes les branches coupées ont été ramassées, séchées et brûlées hors de la parcelle. De même, celles abandonnées dans le verger par les producteurs au cours des opérations d'élagage passées ou tous bois morts accidentellement, par une attaque des insectes, ou par maladies d'origines diverses ont également été systématiquement ramassés et brûlés hors de la plantation. Ces mesures prophylactiques sont appliquées également à toutes les plantations du voisinage dans un rayon de $1-2 \mathrm{~km}$ autour de la plantation-cible afin d'éviter des flux migratoires de $A$. terebrans dans la plantation-cible. Lorsque la plantation couvre une superficie de plus de 5 ha, une bande de 1-2 ha est délimitée pour servir d'unité d'échantillonnage. La mise en place des traitements a eu lieu en août 2015 et la collecte des données a démarré en septembre 2015 et s'est poursuivie jusqu'à la récolte des noix d'anacarde en mars 2016. C'est au cours de cette période de l'année que l'infestation des anacarderaies par $A$. terebrans est habituellement manifeste (Agboton et al., 2017). Les vergers d'anacardiers sans mesures prophylactiques, ont été utilisés comme témoin. Les deux anacarderaies sélectionnées dans une commune sont affectées au hasard à l'un ou l'autre des deux traitements.

\section{Collecte des données :}

Infestation des anacarderaies par A. terebrans: Le taux d'infestation par $A$. terebrans a été déterminé mensuellement en se basant sur le nombre de 'trous d'entrée actifs', c'est-à-dire ceux d'où sortait encore de la sciure de bois, attestant de la présence du ravageur (Agboton et al., 2017). Pour ce faire, à chaque mois d'échantillonnage, 30 arbres sont choisis au hasard et minutieusement inspectés pour la présence de trous d'entrée actifs. Ainsi, le taux d'infestation des vergers inspectés est calculé en rapportant le nombre d'arbres présentant de 'trous d'entrée actifs' par le nombre d'arbres inspectés fois 100 .

Densité de population de A. terebrans dans les anacarderaies : L'estimation de la densité du foreur a été faite mensuellement en utilisant le nombre de trous

\section{RESULTATS}

Taux d'infestation de $A$. terebrans dans les anacarderaies: La figure 1 présente l'évolution temporelle des taux d'infestation des anacardiers par $A$. terebrans dans chaque traitement, respectivement pour les sites de N'dali (Figure 1a), Parakou (Figure 1b) et Tchaourou (Figure 1c). A la mise en place de l'essai en août, le niveau d'infestation des anacarderaies était de zéro sur tous les trois sites (non mentionné sur les actifs observés sur chacun des 30 arbres de l'échantillon utilisé pour déterminer le taux d'infestation. II a été procédé ainsi à cause du caractère cryptique (i.e., caché) de la présence du ravageur. En effet, forant à l'intérieur de l'arbre, aucune observation visuelle du ravageur n'est possible et, par conséquent, une évaluation véritablement exhaustive de sa densité était impossible. Pour contourner cette difficulté, le nombre de trous d'entrée sur un arbre a été assimilé au nombre minimal de foreurs ayant infesté cet arbre, en considérant qu'une galerie abrite (ou a abrité) au minimum un foreur (Agboton et al., 2017). Ainsi, le nombre de trous actifs par arbre a été utilisé pour déterminer la 'densité actuelle' du ravageur c'est-à-dire, celle présente au jour d'échantillonnage ; le nombre de trous récents (i.e. âgés de moins de 3 mois) par arbre permet de déterminer la 'densité récente' du ravageur, c'est-à dire, la densité acquise par le ravageur durant les 3 derniers mois mais au cours de cette même saison; et enfin, le nombre de trous anciens par arbre permet déterminer la 'densité antérieure', correspondant à la densité atteinte par $A$. terebrans au cours de la saison précédente (i.e. au cours des 12 derniers mois), et donc de retracer l'historique de l'attaque de cette plantation par le ravageur.

Mesure de l'effet du traitement sur la productivité des anacarderaies : Pour ce faire, chaque plantation a été subdivisée en trois sous-parcelles abritant à peu près le même nombre d'arbres, et au sein de chaque sous-parcelle 10 arbres ont été choisis au hasard. Les noix produites par les 10 arbres de chaque parcelle ont été récoltées, séchées et stockées dans un même sac jusqu'à la fin de la récolte, et pesée.

Analyses statistiques: Les données ont été analysées par la procédure GLM (General Linear Model) de SAS (2009) après transformation par le logarithme $(\log (X+1)$ et Arcsine $\sqrt{ }($ percent $x / 100)$, respectivement pour les nombres et les pourcentages, et les moyennes sont discernées en utilisant le test de Student-Newman-Keuls au seuil de $a=0,05$.

figures). Pour l'ensemble des trois sites, les taux d'infestation de $A$. terebrans ont été significativement différents et entre les deux traitements $(\mathrm{dl}=1 ; F=$ $17,51 ; P<0,0001)$, et ont varié significativement entre les mois d'échantillonnage $(\mathrm{dl}=6 ; F=17,14 ; P<$ $0,0001)$. En revanche, les différents sites ( $\mathrm{dl}=2 ; F=$ 1,$68 ; P=0,1860$ ), ainsi que l'interaction entre ces sites et les traitements $(\mathrm{dl}=2 ; F=0,04 ; P=0,9603)$ n'ont 
eu aucun effet significatif sur le taux d'infestation de $A$. terebrans dans les anacarderaies. Les résultats par site se présentent comme suit:

Site de N'dali : Sur ce site, le verger le plus infesté a été celui soumis au traitement témoin, avec un taux d'infestation moyen de 40,0 $\pm 9,1 \%$ contre $23,33 \pm$ $7,85 \%$ pour le verger soumis aux mesures prophylactiques. Les pics d'infestation ont été observés en Décembre et Janvier, respectivement au niveau du verger témoin et du verger soumis aux mesures prophylactiques (Figure 1a). Quant aux taux les plus bas, ils ont été enregistrés en Septembre, Octobre, et Novembre pour le témoin et seulement en Septembre pour le verger avec mesures prophylactiques. L'analyse de la variance a révélé une différence significative entre les deux traitements ( $\mathrm{dl}=1 ; F=4,93 ; P=0,0269$ ), avec un taux d'infestation significativement plus élevé dans le témoin que dans le verger avec mesures prophylactiques. Le taux de réduction de l'infestation en comparaison au verger témoin a été de 41,7\%.

Site de Parakou : Ici aussi le taux d'infestation le plus élevé a été observé dans le verger témoin avec une moyenne de 50,0 $\pm 9,0 \%$, contre $33,0 \pm 8,0 \%$ dans le verger soumis aux mesures prophylactiques. Les pics d'infestation ont été observés en Décembre dans le verger témoin et en Novembre dans le verger avec mesures prophylactiques tandis que les infestations les plus faibles ont été relevées en Février pour le premier et en Septembre, Février et Mars pour le second (Figure 1b). Par ailleurs, les taux d'infestation ont été nuls en Octobre et Mars pour le verger avec mesures prophylactiques. L'analyse de la variance a révélé une différence significative entre les deux traitements $(\mathrm{dl}=1$ $; F=0,95 ; P=0,0112$ ), avec un taux d'infestation significativement le plus élevé dans le verger témoin. Le taux de réduction de l'infestation dans le verger avec mesures prophylactiques a été de 34,0\% par rapport au témoin.

Site de Tchaourou : Le taux d'infestation le plus élevé sur ce site a également été observé dans le verger témoin avec une moyenne de $60,0 \pm 9,0 \%$ contre 43,0 $\pm \quad 9,0 \%$ dans le verger soumis mesures prophylactiques. Les pics d'infestation ont été observés en Décembre dans le témoin et en Novembre dans le verger avec mesures prophylactiques. Quant aux plus faibles taux d'infestation, ils ont été observés en Septembre et Mars dans le témoin et en Octobre, Février et Mars dans le verger avec mesures prophylactiques (Figure 1c). L'analyse de variance a révélé une différence significative entre les deux traitements ( $\mathrm{dl}=1 ; F=4,61 ; P=0,03$ ), le taux d'infestation étant significativement le plus élevé dans le verger témoin. Le taux de réduction de l'infestation dans le verger avec mesures prophylactiques a été de $28,33 \%$ en comparaison au verger témoin. 

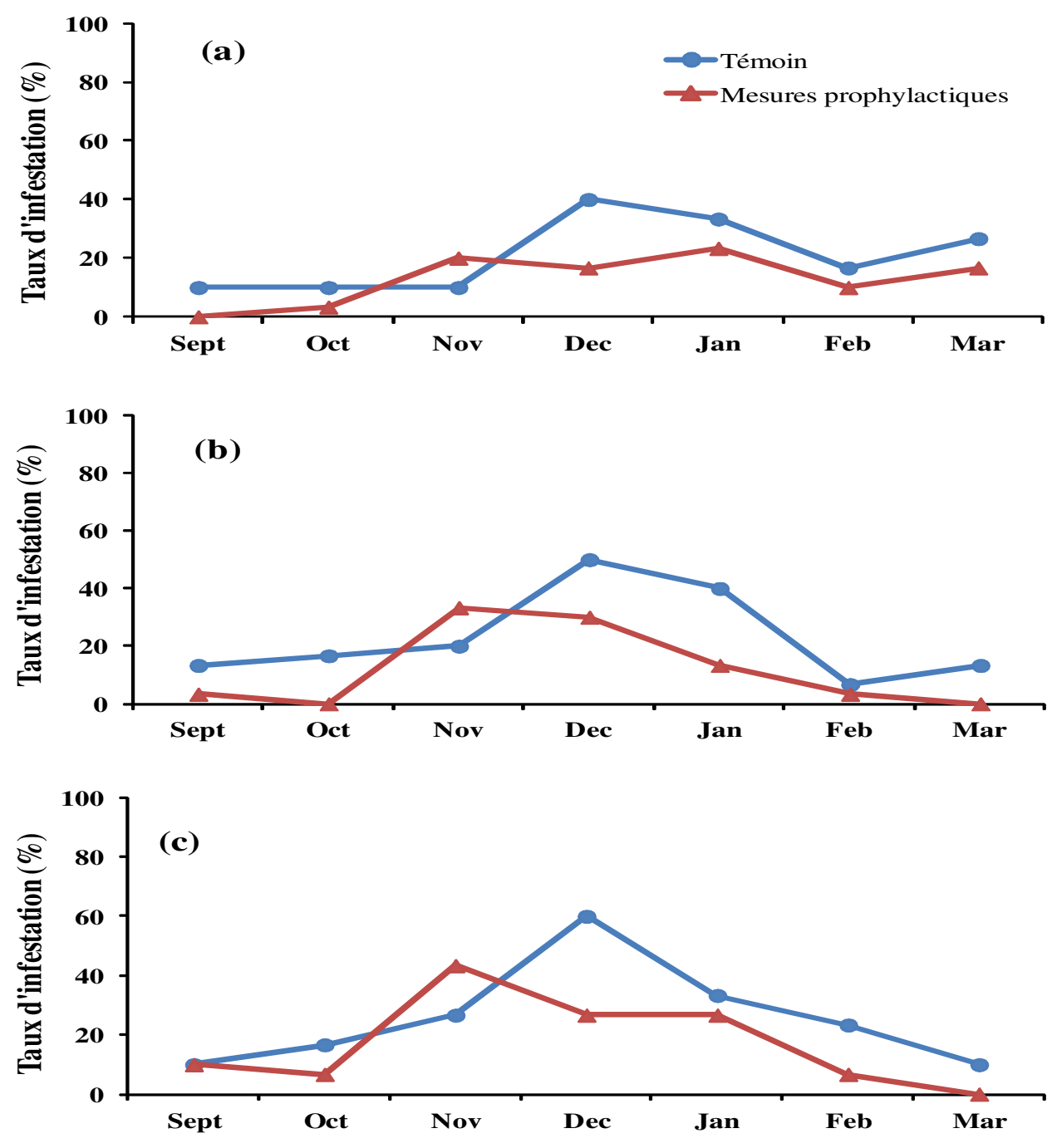

Mois (2015-2016)

Figure 1 : Evolution temporelle du taux d'infestation des anacarderaies par A. terebrans sur le site de : (a) N'dali ; (b) Parakou et (c) Tchaourou.

Evolution des densités de population de $A$. terebrans par traitement et par site : Les figures 2, 3 et 4 présentent l'évolution mensuelle des 'densités actuelles', 'densités récentes' et 'densités antérieures' atteintes par $A$. terebrans au niveau des vergers témoins et vergers avec mesures prophylactiques ainsi que par site pendant la période d'étude.

Densités actuelles' estimées sur la base du nombre des trous actifs : L'analyse de variance de l'ensemble des trois sites a révélé que les traitements $(\mathrm{dl}=1 ; F=$ $22,32 ; P<0,0001)$, les mois d'échantillonnage $(\mathrm{dl}=6$; $F=20,85 ; P<0,0001)$, ainsi que l'interaction entre mois et traitements ( $\mathrm{dl}=6 ; F=7,91 ; P<0,0001)$, ont tous eu un effet significatif sur la 'densité actuelle' de $A$. terebrans. En revanche, ni l'interaction entre sites et traitements (dl $=2 ; F=0,01 ; P=0,9894)$, ni l'interaction entre sites et mois d'échantillonnage $(\mathrm{dl}=$ $12 ; F=1,68 ; P=0,0655)$, n'ont eu d'effets significatifs sur la 'densité actuelle' de $A$. terebrans dans les anacarderaies échantillonnées. Les résultats par site se présentent comme suit :

Site de N'dali: La 'densité actuelle' moyenne de $A$. terebrans a varié mensuellement entre 0,56 $\pm 0,31$ et $6,66 \pm 2,09$ individus par arbre dans le traitement 

dans la lutte contre le foreur de bois, Apate terebrans, dans les anacarderaies du Nord-Bénin

témoin, et entre $0,00 \pm 0,00$ et $4,23 \pm 2,02$ individus par arbre dans celui soumis aux mesures prophylactiques. Les pics ont été observés en Décembre et en Novembre, respectivement dans le verger témoin et celui avec mesures prophylactiques (Figure 2a). Les densités moyennes cumulées tout au long de la période d'échantillonnage ont été de 15,43 \pm 6,05 et de $6,90 \pm 3,29$ individus par arbre, respectivement dans le verger témoin et celui avec mesures prophylactiques, soit un taux de réduction de densité de $55,28 \%$ dans le verger soumis aux mesures prophylactiques. L'analyse de variance a révélé que la 'densité actuelle' de $A$. terebrans a été significativement la plus élevée dans le verger témoin ( $\mathrm{dl}=1 ; F=8,68$; $P=0,0034$ ).

Site de Parakou : La 'densité actuelle' moyenne de $A$. terebrans a varié mensuellement entre $0,33 \pm 0,18$ et $9,46 \pm 2,52$ individus par arbre dans le verger témoin, et entre $0,0 \pm 0,0$ et $5,30 \pm 1,88$ individus par arbre dans celui soumis aux mesures prophylactiques. Les pics ont été observés en Décembre dans les deux vergers (Figure $2 b$ ). Les densités moyennes cumulées tout au long de la période d'échantillonnage ont été de $21,00 \pm 6,88$ et de 10,64 $\pm 4,13$ individus par arbre, dans le verger témoin et celui avec mesures prophylactiques, soit un taux de réduction de $49,33 \%$ dans le verger avec mesures prophylactiques en comparaison au verger témoin. L'analyse de variance a révélé que la 'densité actuelle' de $A$. terebrans a été significativement la plus élevée dans le verger témoin (dl $=1 ; F=5,83 ; P=0,0161$ ).

Site de Tchaourou : La 'densité actuelle' moyenne de A. terebrans a varié mensuellement entre $0,76 \pm 0,33$ et $10,73 \pm 2,10$ individus par arbre dans le verger témoin, et entre $0,10 \pm 0,10$ et $6,83 \pm 2,10$ individus par arbre dans celui soumis aux mesures prophylactiques, avec, respectivement des pics en Décembre et en Novembre (Figure 2c). Les densités moyennes cumulées tout au long de la période d'échantillonnage ont été de $23,34 \pm 7,14$ et de 13,9 \pm 5,25 individus par arbre, respectivement dans le verger témoin et celui avec mesures prophylactiques. Le taux de réduction de densité a été de $40,45 \%$ dans le verger soumis aux mesures prophylactiques en comparaison au verger témoin. L'analyse de variance a révélé que, la 'densité actuelle' de $A$. terebrans a été significativement la plus élevée dans le verger témoin (dl $=1 ; F=5,82 ; P=0,0163$ ).

Densités récentes' estimées sur la base du nombre de trous récents: L'analyse de variance pour l'ensemble des trois sites a révélé que seul le mois d'échantillonnage a eu un effet significatif sur la 'densité récente' de $A$. terebrans ( $\mathrm{dl}=6 ; F=7,43 ; P<0,0001)$. En revanche, ni les sites ( $\mathrm{dl}=2 ; F=0,44 ; P=0,6464)$ qui ont abrité les champs d'expérimentation, ni les différents traitements ( $\mathrm{dl}=1 ; F=1,81 ; P=0,1784)$, ni les interactions entre ces sites et les traitements ( $\mathrm{dl}=2$ ; $F=0,97 ; P=0,3794$ ), ou entre les mois et les traitements ( $\mathrm{dl}=6 ; F=0,56 ; P=0,7634)$ ou encore entre sites et mois ( $\mathrm{dl}=12 ; F=0,21 ; P=0,9982$ ) n'ont eu un effet significatif sur la 'densité récente' de $A$. terebrans dans les anacarderaies échantillonnées. Les résultats par site se présentent comme suit :

Site de N'dali : La 'densité récente' moyenne de $A$. terebrans, a varié mensuellement entre $0,16 \pm 0,13$ et $1,93 \pm 0,51$ individus par arbre dans le verger témoin, et entre $0,3 \pm 0,16$ et $1,43 \pm 0,58$ individus par arbre dans celui soumis aux mesures prophylactiques. Les pics ont été observés en Octobre, Novembre et Février dans le verger témoin et en Octobre et Novembre dans le verger avec mesures prophylactiques (Figure 3a). Les densités moyennes cumulées pendant la période d'échantillonnage, ont été de $9,13 \pm 2,95$ et de $5,7 \pm 2,45$ individus par arbre, respectivement dans le verger témoin et dans le verger sujet aux mesures prophylactiques. L'analyse de variance a révélé que la 'densité récente' de $A$. terebrans a été significativement la plus élevée dans le verger témoin ( $\mathrm{dl}=1 ; F=3,95 ; P=0,0475)$.

Site de Parakou : La 'densité récente' moyenne de $A$. terebrans a varié mensuellement entre $0,46 \pm 0,22$ et $2,50 \pm 0,87$ individus par arbre pour dans le verger témoin, et entre $0,16 \pm 0,16$ et 2,66 $\pm 0,93$ individus par arbre dans le verger soumis aux mesures prophylactiques avec des pics observés respectivement en Novembre et en Décembre (Figure 3b). Les densités moyennes cumulées tout au long de la période d'échantillonnage, ont été de 8,43 $\pm 3,06$ et de 9,10 \pm 3,6 individus par arbre, respectivement dans le verger témoin et dans le verger soumis aux mesures prophylactiques. L'analyse de variance n'a révélé aucune différence significative entre les deux vergers (dl $=1 ; F=0,13 ; P=0,7155)$.

Site de Tchaourou: Ici, la 'densité récente' moyenne de $A$. terebrans a varié entre $0,56 \pm 0,37$ et $2,33 \pm 0,91$ individus par arbre dans le verger témoin et entre 0,56 $\pm 0,24$ et 2,16 $\pm 0,73$ individus par arbre dans le verger soumis aux mesures prophylactiques, avec des pics observés en Novembre dans le verger témoin et en Octobre et Novembre dans le second (Figure 3c). Les densités moyennes cumulées tout au long de la période d'échantillonnage, ont été de 10,63 $\pm 4,86$ et de $8,76 \pm$ 
Onzo et al., J. Appl. Biosci. 2018 Efficacité du ramassage et du brûlage systématiques des bois morts dans la lutte contre le foreur de bois, Apate terebrans, dans les anacarderaies du Nord-Bénin

3,24 individus par arbre, respectivement dans le verger aucune différence significative entre les deux vergers témoin et dans le verger soumis aux mesures $(\mathrm{dl}=1 ; F=0,00 ; P=0,9550)$.

prophylactiques. L'analyse de variance n'a révélé
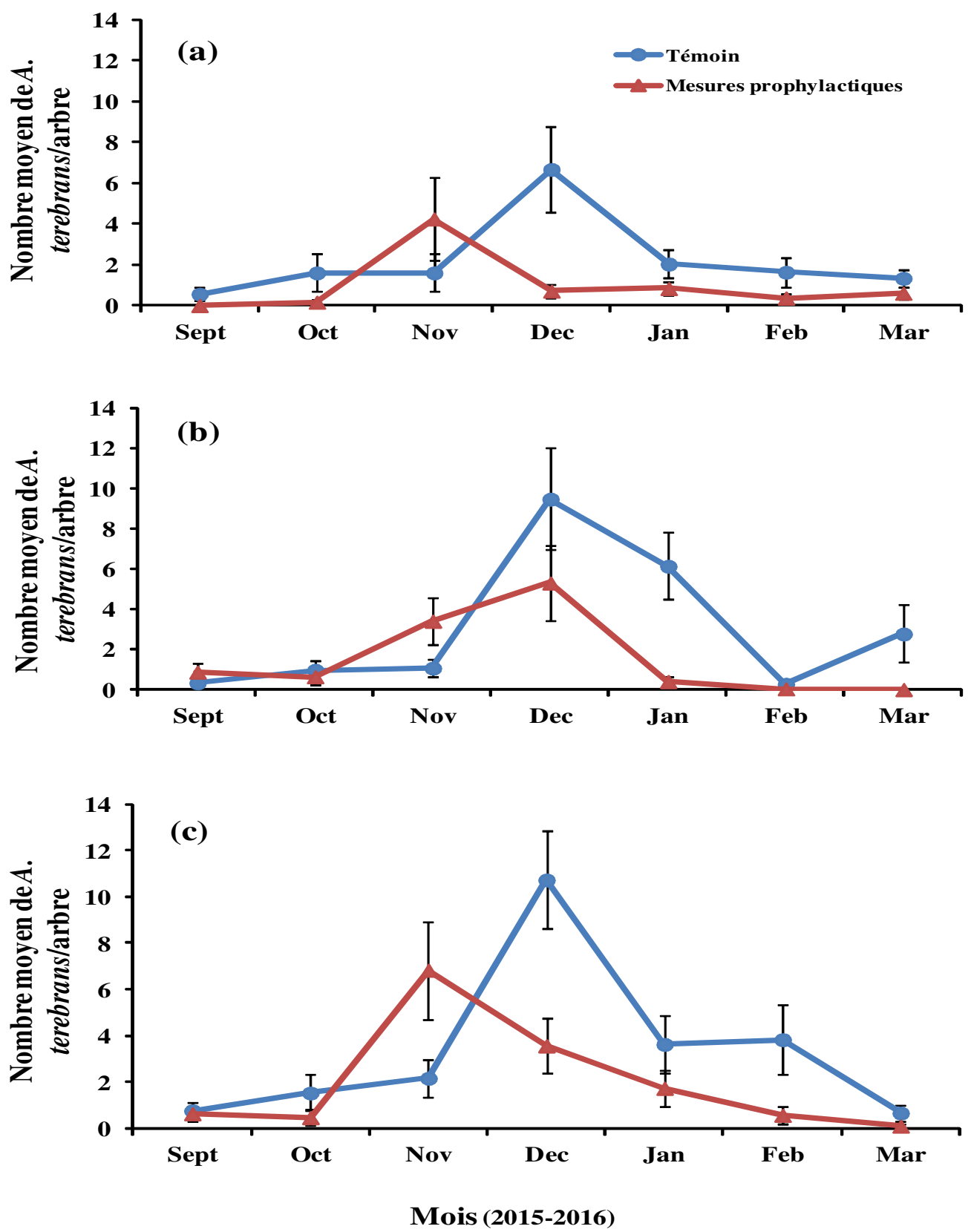

Figure 2: Evolution temporelle de la 'densité actuelle' de A. terebrans dans les anacarderaies sur le site de : (a) N'dali ; (b) Parakou et (c) Tchaourou. 

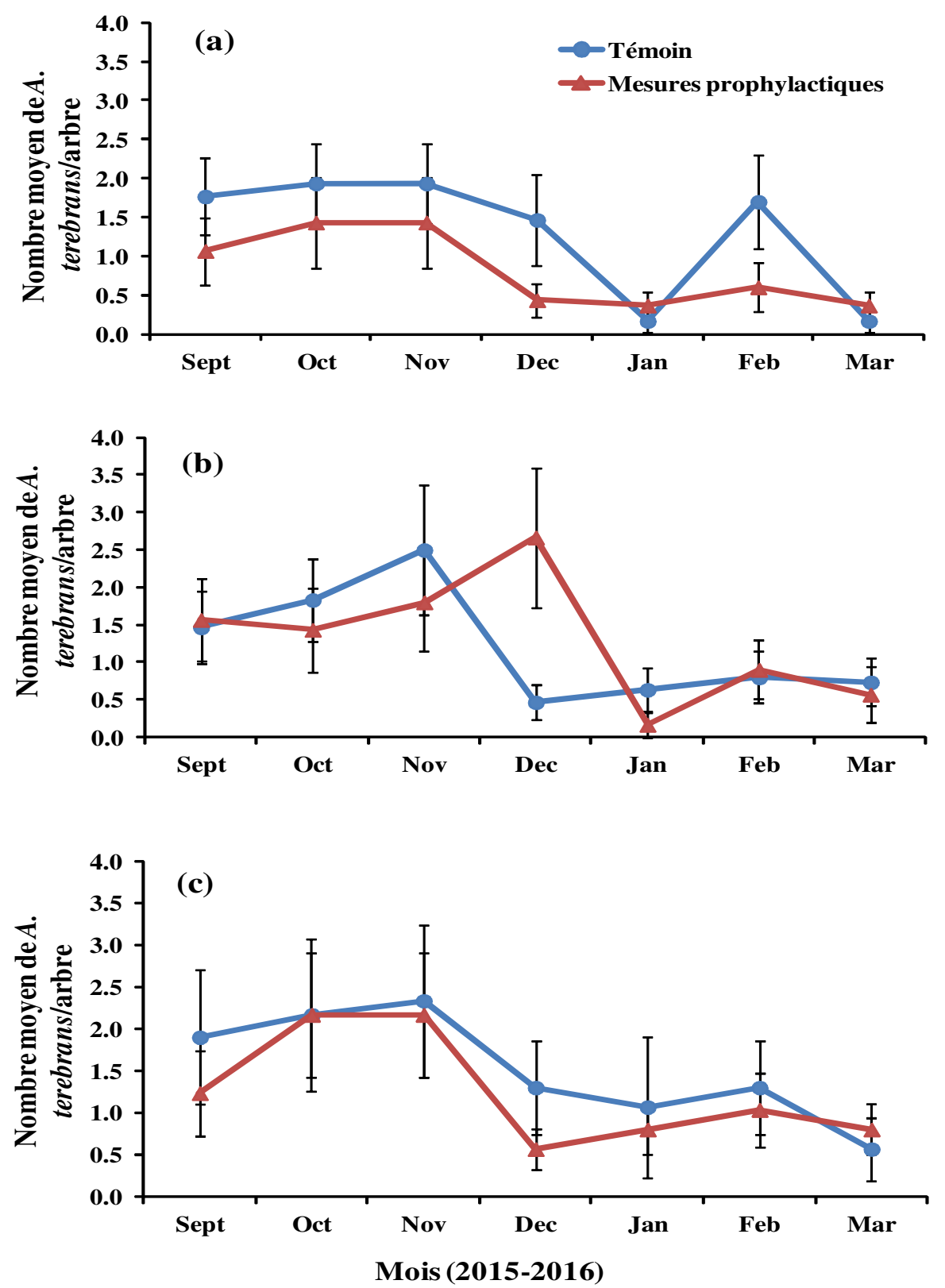

Figure 3: Evolution temporelle de la 'densité récente' de A. terebrans dans les anacarderaies sur le site de : (a) N'dali ; (b) Parakou et (c) Tchaourou. 

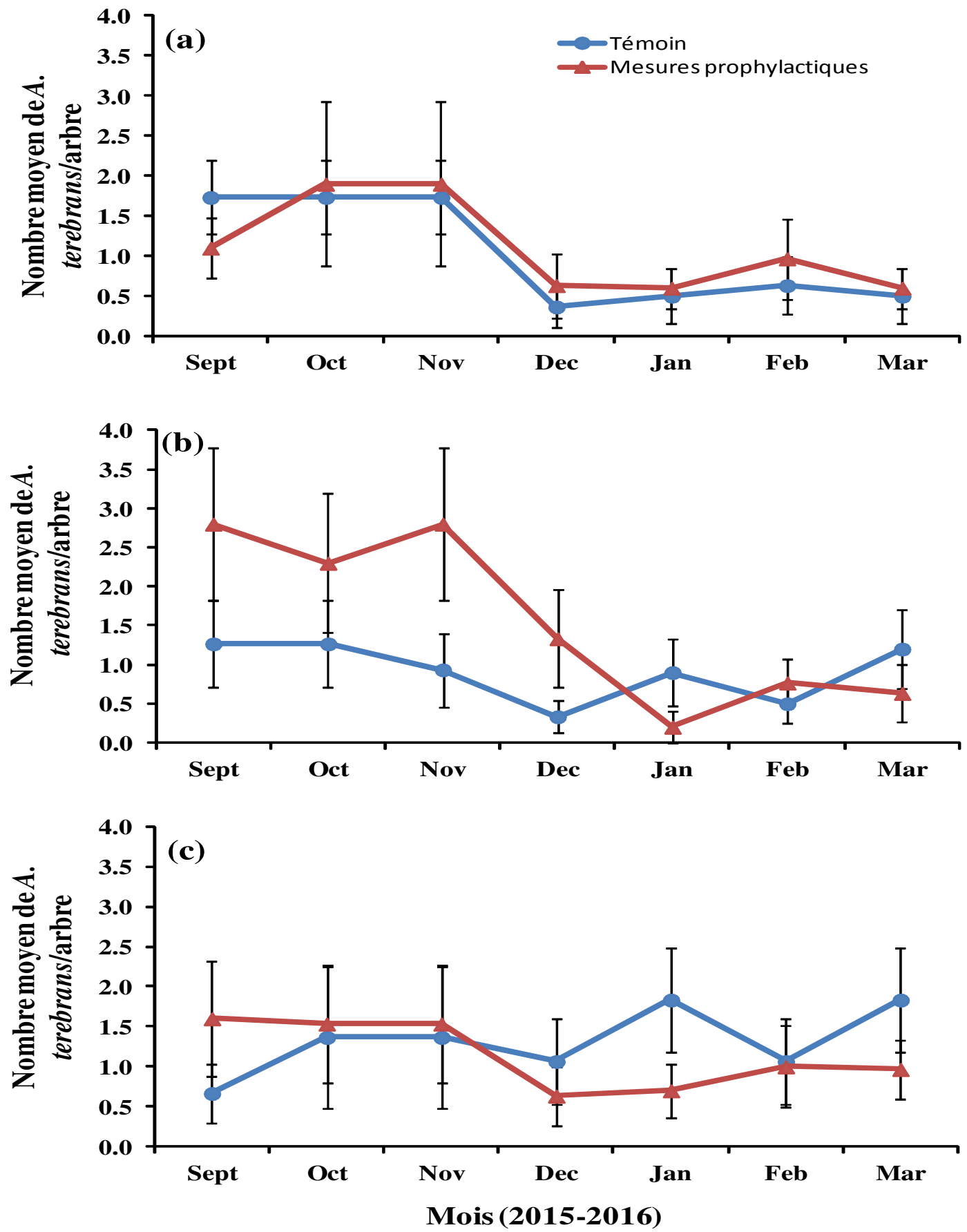

Figure 4: Evolution temporelle de la 'densité antérieure' de A. terebrans dans les anacarderaies sur le site de : (a) N'dali ; (b) Parakou et (c) Tchaourou.

Densités antérieures' estimées sur la base du nombre de trous anciens: A N'dali, la 'densité antérieure' moyenne de A. terebrans (i.e. densité réalisée au cours de la saison dernière) a varié mensuellement entre $0,36 \pm 0,25$ et $1,73 \pm 0,46$ individus par arbre dans le verger témoin et entre 0,60 $\pm 0,24$ et 1,90 $\pm 1,02$ individus par arbre dans le verger soumis aux mesures prophylactiques, avec des pics 
observés en Septembre, Octobre et Novembre dans le témoin, et en Octobre et Novembre dans le verger avec mesures prophylactiques (Figure 4a). A Parakou, la 'densité antérieure' moyenne de $A$. terebrans a varié mensuellement entre $0,33 \pm 0,19$ et $1,26 \pm 0,55$ individus par arbre dans le témoin, et entre $0,20 \pm 0,20$ et 2,80 $\pm 0,98 A$. terebrans par arbre dans le verger avec mesures prophylactiques. Les pics ont été enregistrés en Novembre dans le verger avec mesures prophylactiques et en Octobre, Janvier et Mars dans le verger témoin (Figure $4 b$ ). A Tchaourou, la 'densité antérieure' moyenne de $A$. terebrans a varié mensuellement entre $0,66 \pm 0,36$ et $1,83 \pm 0,64 A$. terebrans par arbre dans le verger témoin et entre 0,63 $\pm 0,37$ et $1,60 \pm 0,72$ individus par arbre dans le verger avec mesures prophylactiques. Les pics ont été enregistrés en Janvier et Mars dans le témoin, contre Septembre et Février dans le verger avec mesures prophylactiques (Figure 4c). L'analyse de variance de l'ensemble des trois sites révèle qu'à l'exception des mois d'échantillonnage ( $\mathrm{dl}=6 ; F=3,37 ; P=0,0027)$, les sites, les traitements de même que leurs

\section{DISCUSSION}

Cette étude qui est sans nul doute la première réalisée au Bénin et dans la sous-région pour mesurer l'impact des mesures prophylactiques dans la lutte contre le foreur de bois, $A$. terebrans, dans les anacarderaies. Elle fait suite à l'étude effectuée par Agboton et al. (2017) sur la dynamique des populations de ce ravageur dans les anacarderaies du Nord-Bénin et ouvre ainsi des perspectives pour l'amélioration de la productivité des anacarderaies à travers une meilleure gestion de $A$. terebrans. En effet, nos résultats montrent clairement que les taux d'infestation des vergers par $A$. terebrans ainsi que les 'densités actuelles' atteintes par le ravageur sont significativement influencés par le ramassage et brûlage systématiques de tous les bois morts (naturellement) ou coupés présents à l'intérieur ou dans le voisinage des anacarderaies. Ainsi, sur les trois sites couverts par l'étude, il apparait nettement que les taux d'infestation de même que les 'densités actuelles' du ravageur sont significativement plus élevés dans les vergers témoins que dans les vergers soumis aux mesures prophylactiques. Bien que l'amplitude des paramètres mesurés varie d'un site à une autre, il est important de remarquer que l'effet des mesures prophylactiques est positif sur tous les sites, avec des taux de réduction de densité allant de $40,45 \%$ à $55,28 \%$. Nos résultats confirment la pertinence des interactions n'ont eu aucun effet significatif sur la densité antérieurement réalisée par $A$. terebrans.

Mesure de l'impact des différents traitements sur la productivité des anacarderaies : Sur le site de N'dali, les productivités moyennes obtenues ont été de 13,57 $\pm 2,64 \mathrm{~kg}$ et 12,83 $\pm 1,73 \mathrm{~kg}$ par arbre, respectivement dans le verger avec mesures prophylactiques et dans le verger témoin. A Parakou, elles ont été de 10,20 $\pm 1,79$ $\mathrm{kg}$ et 9,37 $\pm 1,03 \mathrm{~kg}$ par arbre, respectivement dans le verger avec et celui sans mesures prophylactiques; et de $11,10 \pm 2,15 \mathrm{~kg}$ et $8,30 \pm 0,87 \mathrm{~kg}$ par arbre, respectivement dans le verger avec et celui sans mesures prophylactiques sur le site de Tchaourou. Les résultats de l'analyse statistique révèlent que les productivités moyennes par arbre ne sont pas significativement différentes entre les deux traitements $(\mathrm{dl}=1 ; F=0,97 ; P=0,3439)$, et que ni le site ( $\mathrm{dl}=2$; $F=2,04 ; P=0,1293)$, ni l'interaction site * traitement (dl $=1 ; F=0,21 ; P=0,8155)$, n'ont eu un effet significatif sur la productivité des anacarderaies sur l'ensemble des trois sites.

observations faites récemment par Agboton et al. (2017), qui ont rapporté que du bois mort ou en décomposition serait un substrat d'élevage ou de nidification préféré pour les larves et pupes de $A$. terebrans. En effet, en dehors du stade adulte que l'on retrouve habituellement, aucun stade juvénile de $A$. terebrans n'a jamais été collecté dans le bois vivant. En revanche, ces stades se retrouvent dans le bois en décomposition où ils évoluent jusqu'à l'imago qui va émerger et alors attaquer l'anacardier vivant (Agboton et al., 2017). Ainsi, pendant que les efforts de recherche se poursuivent pour identifier des ennemis naturels de $A$. terebrans, comme par exemple des prédateurs, des parasitoïdes ou des entomopathogènes susceptibles de limiter les populations de ce ravageur dans les anacarderaies, et par ricochet leur impact sur le rendement en noix des arbres, l'application rigoureuse des mesures prophylactiques, telles que décrites dans la présente étude, pourrait déjà être conseillée aux producteurs sur la base de nos résultats. En effet, une destruction du bois mort conduit à une élimination effective de tous les stades juvéniles du ravageur; ce qui réduit considérablement la population de la prochaine génération du ravageur et donc leur attaque dans les vergers. Ce qui est encore intéressant dans nos résultats est la rapidité de la réponse du ravageur au 
traitement. En effet, pendant que les 'densités antérieures' du ravageur (décrivant le niveau de densité atteinte au cours de la saison passée) ne présentaient aucune différence entre les vergers témoins et ceux avec mesures prophylactiques et sur tous les sites d'expérimentation (Figure 4), la réduction des 'densités actuelles' et des taux d'infestation est sans équivoque après la mise en place des traitements. Néanmoins, il importe de faire remarquer qu'il a fallu un certain temps de latence, après la mise en place du traitement, avant que son impact ne soit manifeste sur l'ensemble des trois sites d'étude. C'est ce qui explique que l'analyse statistique des 'densités récentes' (i.e. densités acquises en début de saison), n'a pas fait ressortir de différence significative entre traitements, sauf sur le site de N'dali, quand bien même ces densités sont relativement plus élevées dans les plantations témoins que dans celles soumises aux mesures prophylactiques. On peut donc déduire de ces observations que, plus longue sera la durée de mise en œuvre des mesures prophylactiques et plus important sera leur impact sur le taux d'infestation et la densité de A. terebrans dans les anacarderaies. Notre étude montre ainsi qu'en gérant convenablement les anacarderaies et les formations ligneuses environnantes par le ramassage et brûlage systématiques des branches mortes et des morceaux de bois en décomposition, il devrait être possible d'éliminer la majeure partie de la source locale d'infestation de ce foreur, comme le prédisaient Agboton et al. (2017).Par ailleurs, nos résultats ont montré sur tous les trois sites d'étude que, bien que pas de manière statistiquement significative, la production en noix des anacardiers était plus élevée dans les plantations ayant fait objet de mesures prophylactiques que dans les plantations témoins. II est

\section{REMERCIEMENTS}

Nous remercions le Programme de Productivité Agricole en Afrique de l'Ouest (PPAAO) pour son appui matériel et financier à la réalisation de cette étude.

\section{REFERENCES}

Afouda LCA, Zinsou V, Balogoun RK, Onzo A, Ahohuendo BC, 2013. Inventaire des agents pathogènes de l'anacardier (Anacardium occidentale L.) au Bénin. Bulletin de la Recherche Agronomique du Bénin 73: 13-19.

Agboton C, Onzo A, Ouessou FI, Goergen G, Vidal S, Tamò M, 2014. Insect fauna associated with Anacardium occidentale (Sapindales: à espérer donc, comme nous le disions plus haut, que plus longue sera la durée d'application de ces mesures prophylactiques et plus important sera leur impact sur le niveau de population de $A$. terebrans et donc sur la productivité des anacarderaies. En effet, l'impact relativement positif observé sur le rendement en noix des anacardiers juste après quelques mois d'application préfigure d'un impact certainement plus significatif, tant au plan qualitatif que quantitatif, du traitement sur la productivité des anacarderaies dès que cette pratique sera bien vulgarisée et adoptée par la majorité des producteurs de noix d'anacarde. Son adoption permettrait également au Bénin et à d'autres pays de la sous-région de continuer par mettre sur le marché international des noix d'anacarde dépourvues de tout résidu de pesticide, pour le bien-être des consommateurs. En conclusion, bien que le ramassage et le brûlage systématiques du bois mort au sein et aux alentours des anacarderaies permettent une réduction sensible de la population du ravageur, la mise en œuvre effective de cette méthode de lutte exige l'adhésion et la participation active de toute la communauté. Aussi, les implications de cette méthode en terme d'une main-d'œuvre conséquente ne devraient pas être occultées, ceci d'autant que le ravageur dispose d'une gamme très élargie de planteshôtes (Agboton et al., 2014). Ainsi donc, bien qu'efficace cette mesure ne saurait être considérée comme le seul moyen de lutte contre $A$. terebrans en négligeant la recherche d'autres moyens, surtout biologiques, de lutte contre ce ravageur à travers une meilleure connaissance de sa biologie. Ceci serait le gage d'une production écologiquement saine et durable de la noix d'anacarde au Bénin et dans la sous-région Ouest-Africaine.

Notre gratitude va également aux producteurs d'anacarde du Nord-Bénin qui ont mis leurs vergers à notre disposition pour la conduite des essais.

Anacardiaceae) in Benin, West Africa. Journal of Insect Science DOI: http://dx.doi.org/ 10.1093/isesa/ieu091.

Agboton C, Onzo A, Korie S, Tamò M, Vidal S, 2017. Spatial and temporal infestation rates of Apate terebrans (Coleoptera: Bostrichidae) in cashew orchards in Benin, West Africa. African Entomology 25(1): 24-36. 
Agboton C, Onzo A, Akohou AH, Goergen G, Vidal S, Tamò M, 2018. Population dynamics of the cashew leafminer, Eteoryctis gemoniella (Lepidoptera: Gracillariidae), and inventory of its parasitoids in cashew orchards of Northern Benin, West Africa. International Journal of Tropical Insect Science, DOI: https://doi.org/10.1017/S1742758417000339.

Asogwa EU, Anikwe JC, Ndubuaku TCN, Okelana FA, 2009. Distribution and damage characteristics of an emerging insect pest of cashew, Plocaederus ferrugineus L. (Coleoptera: Cerambycidae) in Nigeria: a preliminary report. African Journal of Biotechnology 8: 053-058.

Dwomoh EA., Ackonor JB, Afun JVK, 2008. Survey of insect species associated with cashew (Anacardium occidentale Linn.) and their distribution in Ghana. African Journal of Agricultural Research 3: 205-214.

FAO, 2002. Base des données de la FAO 2002. http://faostat.fao.org. Visité le 8 juin 2015.

Gagnon MB, 1998. Etude sur la filière anacarde au Bénin. Rapport définitif, INRAB, Cotonou, Bénin. 85 pages.

Gardner JCM, 1957. An annotated list of East African Forest Insects. East African Agriculture and Forestry Research Organisation (EAAFRO),
Forestry Technical. Notes 7: 1-48. Ouessou IF, 2010. Inventaire de l'entomofaune associée à l'anacardier (Anacardium occidentale Linn.) dans les départements du Centre et du Nord du Bénin. Mémoire d'Ingénieur agronome, Université de Parakou (Bénin), 58 pages.

PADSE, 2004. Gestion des attaques du foreur de bois Apate terebrans (Pallas, 1772) dans les plantations d'anacardier au Bénin, fiche technique. Cotonou. 26 pages.

SAS Institute. 2009. Base SAS ${ }^{\circledR} 9.2$ procedures guide. SAS Institute Inc., Cary, North Carolina, USA.

Topper CP, 2002. Issues and constraints related to the development of cashew nuts from five selected african countries (Côte d'Ivoire, Ghana, Guinea, Guinea Bissau and Nigeria). Cotonou. 24 pages.

Vasconcelos S, Mendes LF, Beja P, Hodgson CJ., Catarino L, 2014. New records of insect pest species associated with cashew, Anacardium occidentale L. (Anacardiaceae), in GuineaBissau. African Entomology 22(3): 673-677.

Wagner MR, Cobbinah JR, Bosu PP, 2008. Forest Entomology in West Tropical Africa: Forest Insects of Ghana. Published by Springer, P.O. Box 17, 3300 AA Dordrecht- The Netherlands. 305 pages. 